\title{
Sex Differences in Selecting Between Food and Cocaine Reinforcement are Mediated by Estrogen
}

\author{
Kerry A Kerstetter*,', Maya A Ballis', Stevie Duffin-Lutgen', Amanda E Carr', Alexandra M Behrens' and \\ Tod E Kippin ${ }^{1,2}$ \\ 'Department of Psychological and Brain Sciences, University of California, Santa Barbara, CA, USA; ${ }^{2}$ Neuroscience Research Institute, \\ University of California, Santa Barbara, CA, USA
}

\begin{abstract}
Cocaine-dependent women, relative to their male counterparts, report shorter cocaine-free periods and report transiting faster from first use to entering treatment for addiction. Similarly, preclinical studies indicate that female rats, particularly those in the estrus phase of their reproductive cycle, show increased operant responding for cocaine under a wide variety of schedules. Making maladaptive choices is a component of drug dependence, and concurrent reinforcement schedules that examine cocaine choice offers an animal model of the conditions of human drug use; therefore, the examination of sex differences in decision-making may be critical to understanding why women display a more severe profile of cocaine addiction than men. Accordingly, we assessed sex and estrous cycle differences in choice between food (45 mg grain pellets) and intravenous cocaine $(0.4 \mathrm{or} 1.0 \mathrm{mg} / \mathrm{kg}$ per infusion) reinforcement in male, female (freely cycling), and ovariectomized $(\mathrm{OVX})$ females treated with either estrogen benzoate (EB; $5 \mu \mathrm{g}$ per day) or vehicle. At both cocaine doses, intact female rats choose cocaine over food significantly more than male rats. However, the estrous cycle did not impact the level of cocaine choice in intact females. Nevertheless, OVX females treated with vehicle exhibited a substantially lower cocaine choice compared with those receiving daily EB or to intact females. These results demonstrate that intact females have a greater preference for cocaine over food compared with males. Furthermore, this higher preference is estrogen-dependent, but does not vary across the female reproductive cycle, suggesting that ovarian hormones regulate cocaine choice. The present findings indicate that there is a biological predisposition for females to forgo food reinforcement to obtain cocaine reinforcement, which may substantially contribute to women experiencing a more severe profile of cocaine addiction than men.

Neuropsychopharmacology (2012) 37, 2605-26I4; doi:I0.1038/npp.2012.99; published online 8 August 2012
\end{abstract}

Keywords: sex differences; cocaine; self-administration; choice; ovariectomy; estrogen

\section{INTRODUCTION}

Clinical and preclinical studies indicate that there are sex differences in the response to cocaine and in the cocaineaddiction process (see Anker and Carroll, 2010; Becker, 2009). Women, relative to men, transition faster from first cocaine use to entering treatment (Westermeyer and Boedicker, 2000), report shorter cocaine-free periods (Kosten et al, 1993), and experience greater cocaine craving in response to psychological and physical stressors (Back et al, 2005). Similarly, female rats, relative to males, display a greater propensity to acquire cocaine self-administration (Lynch and Carroll, 1999), enhanced cocaine self-administration under extended access (Roth and Carroll, 2004), and progressive ratio schedules (Lynch and Taylor, 2004).

*Correspondence: Dr KA Kerstetter, Center for Integrative Brain Research, Seattle Children's Hospital, Seattle, WA 98101, USA, Tel: + | 206884 | |38, Fax: + | 206884 1210,

E-mail: kerry.kerstetter@seattlechildrens.org

Received 30 March 20 I I; revised I5 May 20 I2; accepted 2 I May 2012
However, it is unknown if there are sex differences in the propensity to choose cocaine over natural reinforcers.

The subjective and behavioral effects of cocaine fluctuate across the reproductive cycle in females. For women, the positive effects of cocaine administration are greater during the follicular compared with the luteal phase, and these effects are diminished by progesterone administration (eg, Evans et al, 2002; Evans and Foltin, 2006; Sofuoglu et al, 1999). Consistent with the human literature, there is evidence that the estrous cycle modulates cocaine-related behavior in rats. Female rats in the estrus phase of the estrous cycle display greater cocaine seeking relative to non-estrus females (Kippin et al, 2005; Kerstetter et al, 2008; Roberts et al, 1989). Furthermore, ovariectomized (OVX) females exhibit decreased cocaine intake and lower rates of acquisition, relative to OVX females treated with estrogen (Lynch et al, 2001; Larson et al, 2007; Zhao and Becker, 2010), Thus, on several measures, ovarian hormones appear to mediate sex differences in cocaine taking and seeking.

Concurrent reinforcement procedures are important to the understanding of reinforcement value of a drug, because 
they measure the relative strengths of reinforcers in a response-rate-independent fashion (Banks et al, 2008) and have increased clinical relevance compared with single reinforcer procedures (Katz, 1990). Cocaine-dependent humans (mostly male) allowed the opportunity to obtain money or intravenous (i.v.) cocaine infusions will choose cocaine over a small monetary reinforcer (Donny et al, 2003); however, when the monetary reinforcer is increased, the choice can be shifted away from cocaine (Higgins et al, 1994). Similarly, primates choose between cocaine and food reinforcement in a fashion that is sensitive to reinforcer values. Specifically, when the cocaine dose is increased, male rhesus monkeys show a preference for cocaine over food (Banks and Negus, 2010); and when the value of cocaine is diminished by giving non-contingent amphetamine before testing, rhesus monkeys will shift their preference from cocaine to food (Negus, 2003).

Self-administration procedures that provide the choice between two reinforcers during the same session have also been examined in male rats, but have revealed that non-drug reinforcers are powerful alternatives to cocaine. Specifically, most males given the choice between cocaine $(0.25-1.5 \mathrm{mg})$ and sucrose reinforcement during a discrete trials procedure almost exclusively prefer sucrose (Cantin et al, 2010), even following an extensive history of cocaine intake (Lenoir $\mathrm{et} \mathrm{al}$, 2007), suggesting that high cocaine choice offers a unique opportunity to identify addiction vulnerable individuals independent of extensive experience with cocaine (Ahmed, 2010). In addition, although sex differences in choice behavior have not been studied extensively, one study reported that females reduced their cocaine self-administration significantly more than males when a running wheel was concurrently available (Cosgrove et al, 2002).

Although not previously examined, the extensive evidence indicating sex differences in response to cocaine suggests that females will exhibit higher cocaine preferences than males when given a choice to respond for cocaine or food reinforcement. Making maladaptive choices is a large component of drug dependence and concurrent reinforcement schedules that examine cocaine choice offers an animal model of the conditions of human drug use; therefore, the examination of sex differences in decisionmaking may be critical to understanding why women display a more severe profile of cocaine addiction than men. Accordingly, the present study provides the first examination of the effects of sex, ovariectomy, and estrogen on choice between cocaine and food. Importantly, our results indicate that female rats place a higher preference on cocaine than do male rats, and that this difference is ovarian hormone-dependent.

\section{MATERIALS AND METHODS}

\section{Subjects}

Male ( $n=39$, weighing between 275 and $300 \mathrm{~g}$ ) and female ( $n=44$, weighing between 225 and $250 \mathrm{~g}$ ) Sprague-Dawley rats (Charles-River, Wilmington, MA) were individually housed in a temperature- and humidity-controlled vivarium on a 12-h light-dark cycle. In most experiments, rats' access to food (outside experimental sessions) was restricted to $20 \mathrm{~g}$ for females and $25 \mathrm{~g}$ for males of rat chow per day (Harlan,
Indianapolis, IN); an additional set of rats was maintained on ad libitum rat chow (males: $n=7$, females: $n=5$ ). All rats were maintained on ad libitum water access. The housing and care of the rats followed the "Guidelines for the Care and Use of Mammals in Neuroscience and Behavioral Research' (National Research Council 2003) and was approved by the Institutional Animal Care and Use Committee.

\section{Ovariectomy and Catheter Surgery}

Sixteen of the females underwent bilateral ovariectomy (performed by the vendor) 2 weeks before catheter implantation. Male and female rats were anesthetized via isoflourane gas ( $5 \%$ for induction; $2.5 \%$ for maintenance), and a jugular catheter was implanted as previously described (Kerstetter et al, 2008). Chronic indwelling catheters were constructed using a bent steel cannula with a screw-type connector (Plastics One, Roanoke, VA), silastic tubing $(10 \mathrm{~cm}$, i.d. $0.64 \mathrm{~mm}$, o.d. $1.19 \mathrm{~mm}$; Dow Corning, Midland, MI), polypropylene mesh (Atrium Medical, Hudson, NH), and cranioplastic cement. The catheter was inserted into the right jugular vein, secured to surrounding tissue, and then ran subcutaneously to exit posterior to the shoulder blades. All rats were allowed a minimum of 5 days to recover from surgery before operant training.

Following surgery, catheters were flushed before operant sessions with ticarcillin disodium/clavulanate potassium (Timentin; $10 \mathrm{mg} / 0.1 \mathrm{ml}$; Schein Pharmaceutical, Florham Park, NJ) dissolved in $0.9 \%$ physiological saline and $0.1 \mathrm{ml}$ of heparin after self-administration sessions $(6.0 \mathrm{IU} / 0.1 \mathrm{ml}$ prepared in $0.9 \%$ physiological saline, i.v.) as a prophylactic measure against microbial infection and to extend catheter patency. Catheter patency was verified by infusing 0.12 (males) or $0.04 \mathrm{ml}$ (females) of methohexital sodium $(10 \mathrm{mg} / \mathrm{ml}$ i.v.; Eli Lilly, Indianapolis, IN), which produces a rapid loss of muscle tone only when administered intravenously.

\section{Estrous Cycle Monitoring}

Estrous phase determination was based on vaginal smears collected before operant sessions as described previously (Kerstetter et al, 2008). Samples were collected using a gentle sweeping motion with a sterile, saline-dipped, cottontipped applicator, and smeared onto a glass slide. Smears were stained with Giemsa (Sigma-Aldrich, St Louis, MO) and cell morphology was assessed under a light microscope at $\times 10$ magnification. The metestrus (also known as diestrus I) phase was defined as the presence of approximately equal proportions of nucleated epithelial cells, non-nucleated cornified epithelial cells, and leukocytes. The diestrus (also known as diestrus II) phase was defined as a minimum amount of cells, including leukocytes and occasional epithelia. The proestrus phase was defined by the presence of more than $75 \%$ nucleated epithelial cells. The estrus phase was defined as the presence of more than $75 \%$ non-nucleated cornified epithelial cells.

\section{Estrogen Treatments}

OVX females were subcutaneously treated daily with either peanut oil $(n=8$, vehicle, $0.1 \mathrm{ml})$ or estrogen benzoate $(n=8, \mathrm{~EB} ; 5 \mu \mathrm{g} / 0.1 \mathrm{ml}$ peanut oil) $30 \mathrm{~min}$ before operant 
sessions. This dose of EB was selected, because it has been shown to enhance cocaine intake relative to controls in OVX female rats (eg, Zhao and Becker, 2010). Vehicle and EB treatment began on the first day of operant training.

\section{Operant Procedures}

The operant chambers were equipped with two retractable levers, a stimulus light above each lever, a food pellet dispenser between the levers, a white houselight on the wall opposite to the levers, and a speaker connected to a tone generator (ANL-926, Med Associates). Cocaine self-administration and food reinforcement training was conducted during daily 1-h (45 mg pellets; Noyes, Lancaster, $\mathrm{NH}$ ) and 3-h (cocaine hydrochloride; National Institute on Drug Abuse, Research Triangle Park, NC) sessions.

Rats were trained to lever press on the right lever for food and on the left lever for cocaine on alternating days for a minimum of 10 days. The first day of training was counterbalanced for food or cocaine between subjects. At the start of each session, the rat's catheter was connected to a liquid swivel (Instech, Plymouth Meeting, PA) via polyethylene 20 tubing that was encased in a steel spring leash (Plastics One), and the swivel was suspended above the operant conditioning chamber and connected to an infusion pump (Model PHM100, Med Associates). For training sessions, only one lever was extended (ie, only cocaine or food available), and rats were trained to press the lever for reinforcement under a Fixed Interval: $20 \mathrm{~s}$ (FI 20-s) schedule of reinforcement. Accordingly, the training procedures ensured that rats had equal experience in obtaining food and cocaine reinforcement before choice sessions. Throughout the experiment, operant sessions lasted until the rat earned a total of 25 reinforcers, if the rat did not earn 25 reinforcers, the session ended after $3 \mathrm{~h}$ (training and choice sessions).

During food training sessions, responses on the right lever resulted in the delivery of two (females) or three (males) $45 \mathrm{mg}$ grain food pellets into the food pellet dispenser, and a 5-s presentation of the white stimulus light above the food lever. These levels of food reinforcement approximate differences in body weight and were previously shown to produce equivalent operant responding in males and females (Kerstetter et al, 2008b); an additional group of males $(n=6)$ received two pellets to compare the impact of food reinforcement magnitude on cocaine choice. After food pellet delivery, there was a 20 -s interval, during which responding on the lever produced no programmed consequences. Rats had to earn at least 20 reinforcers during a training session to count towards a criterion day. Rats needed five consecutive criterion food self-administration sessions to meet acquisition.

During cocaine training sessions, responses on the left lever resulted in a cocaine infusion that involved a 4-s activation of the infusion pump and a 5-s presentation of the white stimulus light above the cocaine lever. Cocaine hydrochloride was dissolved in saline, filtered using a 0.45$\mu \mathrm{m}$ ultracleaning filter unit (Fisher Scientific), and delivered at a dose of 0.4 or $1.0 \mathrm{mg} / \mathrm{kg}$ per $0.10 \mathrm{ml}$ infusion. These cocaine doses were selected, because they are reinforcing in both male and female rats (see, eg, Caine et al, 2004; Fuchs et al, 2005). After each infusion, there was a 20-s interval, during which responses on the lever produced no programmed consequences. Rats had to earn at least 20 reinforcers during a training session to count towards a criterion day, and rats needed 5 consecutive criterion cocaine self-administration sessions to meet acquisition. Food and cocaine self-administration training continued until the rat had completed acquisition for both food and cocaine (total of 10 criterion training days needed between food and cocaine operant training to meet acquisition criteria).

After training was completed, five concurrent reinforcement sessions (FI 20-s) were conducted, during which both levers were extended, allowing the rat to select either food or cocaine. It was noted that rats responded during the 20-s period when responses were not reinforced, so after the completion of concurrent reinforcement sessions, five discrete trial sessions were conducted under the same conditions as the concurrent reinforcement sessions, with the exception that following a response on either lever, both levers were retracted to prevent non-reinforced responding and returned after an inter-trial interval (ITI) of $20 \mathrm{~s}$, thus minimizing perseveration of response patterns. An additional set of rats (males: $n=7$, females: $n=9$ ) was tested under discrete trial conditions at the $1.0 \mathrm{mg} / \mathrm{kg}$ per infusion cocaine dose, with an ITI of $10 \mathrm{~min}$ and a maximum session time of $360 \mathrm{~min}$. Because of the ITI being extended, the immediate effects of cocaine are presumed to be diminished before the next discrete trial, allowing the assessment of choice with a minimal contribution of the anorexic effects of cocaine. Finally, male and intact female rats underwent 10 reverse discrete trial sessions (ITI 20 s). These sessions were identical to the discrete trial sessions, except now the reinforcer-lever relationship was reversed, such that lever responses on the right lever now lead to cocaine infusions and responses on the left lever led to food pellet delivery.

\section{Data Analysis}

Analyses of variance (ANOVA) were used to analyze days to meet self-administration criteria, lever responses during food and cocaine reinforcement (data collapsed across five criterion sessions for each reinforcer), and lever responses during concurrent reinforcement (last four sessions collapsed) with sex/estrous/OVX status serving as the betweensubjects variables. Furthermore, ANOVA was used to analyze session time (total minutes of session) and percent cocaine choice ((infusions earned/infusions + pellets earned) ${ }^{\star} 100$ ) during concurrent reinforcement (last four sessions collapsed), discrete trials (last four session collapsed), and reverse discrete trials (last five sessions collapsed) with sex/ estrous/hormone status serving as the between-subjects variables. The first session of concurrent and discrete trial sessions and the first five sessions of reverse discrete trials were not included in the analysis, to exclude behavior that was reflective of adjustment to new schedules. The level of statistical significance for all comparisons was 0.05 .

\section{RESULTS}

Sex and Cocaine Dose Effect on the Choice Between Cocaine and Food Reinforcement

Acquisition of operant responses. The number of days to meet criterion for self-administration acquisition for males 
Table I Aquisition (Days to Meet Criteria) and Lever Responses (all Data Represents Mean \pm SEM)

\begin{tabular}{|c|c|c|c|c|c|c|c|c|}
\hline \multirow{2}{*}{$\begin{array}{l}\text { Cocaine dose } \\
\text { (mg/kg i.v.) }\end{array}$} & \multirow[t]{2}{*}{ Sex } & \multirow{2}{*}{$\begin{array}{l}\text { Pellet number } \\
\text { per reinforcer }\end{array}$} & \multirow{2}{*}{$\begin{array}{l}\text { Home-cage } \\
\text { feeding (g) }\end{array}$} & \multirow{2}{*}{$\begin{array}{c}\text { Total acquisition } \\
\text { (days) }\end{array}$} & \multirow{2}{*}{$\begin{array}{l}\text { Responses during } \\
\text { cocaine training }\end{array}$} & \multirow{2}{*}{$\begin{array}{l}\text { Responses during } \\
\text { food training }\end{array}$} & \multicolumn{2}{|c|}{ Concurrent reinforcement } \\
\hline & & & & & & & $\begin{array}{l}\text { Responses on } \\
\text { food lever }\end{array}$ & $\begin{array}{l}\text { Responses on } \\
\text { cocaine lever }\end{array}$ \\
\hline \multirow[t]{2}{*}{0.4} & Male & 3 & 25 & $12.32 \pm 0.23$ & $85.48 \pm 10.70$ & $109.33 \pm 6.83$ & $90.88 \pm 6.99$ & $17.85 \pm 7.17$ \\
\hline & Female & 2 & 20 & $14.55 \pm 0.42^{\mathrm{a}}$ & $56.55 \pm 6.93^{\mathrm{a}}$ & $79.63 \pm 4.91^{\mathrm{a}}$ & $40.23 \pm 6.66^{\mathrm{a}}$ & $21.37 \pm 6.52$ \\
\hline & Female & 2 & 20 & $12.50 \pm 0.26$ & $93.78 \pm 11.72^{\mathrm{a}}$ & $92.45 \pm 5.10^{\mathrm{a}}$ & $35.77 \pm 9.02$ & $29.87 \pm 8.67$ \\
\hline & Male & 3 & Ad libitum & $14.50 \pm 1.59$ & $56.55 \pm 10.82$ & $79.17 \pm 9.43$ & $31.77 \pm 8.72$ & $26.98 \pm 3.63$ \\
\hline & Female & 2 & Ad libitum & $16.60 \pm 1.83$ & $57.09 \pm 5.79$ & $65.56 \pm 5.27$ & $27.52 \pm 9.20$ & $27.72 \pm 2.79$ \\
\hline
\end{tabular}

asignificant effect relative to males under same conditions.

${ }^{\text {b }}$ Significant effect of pellet number per reinforcer in males ps $<0.05$.

Table 2 Session Time (min, all Data Represents Mean \pm SEM)

\begin{tabular}{|c|c|c|c|c|c|}
\hline Cocaine Dose (mg/kg i.v.) & Sex & Cocaine training & Food training & Concurrent reinforcement & Discrete trials \\
\hline \multirow[t]{2}{*}{0.4} & Male & $99.55 \pm 10.23$ & $19.29 \pm 1.99$ & $22.98 \pm 3.69$ & $|5.3| \pm 2.15$ \\
\hline & Female & $90.64 \pm 6.17$ & $18.70 \pm 1.37^{\mathrm{a}}$ & $47.06 \pm 6.41^{\mathrm{a}}$ & $39.19 \pm 5.02^{\mathrm{a}}$ \\
\hline \multirow[t]{2}{*}{ । } & Male & $\mid 45.97 \pm 5.67$ & $31.56 \pm 4.13$ & $90.13 \pm 10.89$ & $73.67 \pm 17.62$ \\
\hline & Female & $125.31 \pm 11.10$ & $22.21 \pm 2.12^{\mathrm{a}}$ & $106.69 \pm 15.79^{\mathrm{a}}$ & $97.73 \pm 17.54^{\mathrm{a}}$ \\
\hline
\end{tabular}

Significant effect relative to males $p<0.05$.

and intact females trained with either 0.4 or $1.0 \mathrm{mg} / \mathrm{kg}$ (i.v.) cocaine were analyzed via a $2 \times 2(\operatorname{sex} \times$ dose $)$ ANOVA, and an interaction of sex and dose was detected $\left(F_{1,61}=14.24\right.$, $p<0.05)$. Follow-up analyses revealed that females took significantly longer ( $\sim 15$ days) than males ( $\sim 12$ days) to reach acquisition when trained on the low dose of cocaine $(p<0.05)$, but no differences between sexes were found when rats were trained on the high dose of cocaine (Table 1).

Cocaine reinforcement during training. Lever responding during the five criterion cocaine self-administration sessions was analyzed via a $2 \times 2(\operatorname{sex} \times$ dose $)$ ANOVA. There was a significant interaction between sex and dose $\left(F_{1,95}=\right.$ $12.86, p<0.05$ ), and follow-up analyses revealed that males responded significantly more than females for the low cocaine dose, but females responded significantly more than males for the high cocaine dose ( $p s<0.05$; Table 1$)$. Additionally, $2 \times 2$ (sex $\times$ dose) ANOVA analysis of average time to obtain 25 reinforcer revealed a significant effect of cocaine dose $\left(F_{1,64}=20.03, p<0.05\right.$; Table 2$)$, with sessions taking longer at the higher cocaine dose. There was no effect of sex or interaction between dose and sex for session time.

Food reinforcement during training. Lever responding during the five criterion food self-administration sessions was analyzed via a $2 \times 2(\operatorname{sex} \times \operatorname{dose})$ ANOVA. There was a significant interaction between sex and dose $\left(F_{1,95}=17.80\right.$, $p<0.05$ ). Responses for food reinforcement exhibited by males and females trained with the low dose of cocaine was analyzed via a one-way ANOVA, which revealed a significant effect of sex with males pressing more than females $\left(F_{1,55}=5.56, p<0.05\right.$; Table 1$)$. Responses for food reinforcement exhibited by males and females trained with the high cocaine dose was analyzed via one-way ANOVA, which revealed a significant effect of sex with females pressing more than males $\left(F_{1,40}=11.17, p<0.05\right.$; Table 1$)$. Females exhibited higher responding for both food and cocaine reinforcement when trained at the higher cocaine dose, whereas males responded more for both reinforcers when trained at the lower cocaine dose. Session time was analyzed via a $2 \times 2($ sex $\times$ dose $)$ ANOVA. There was a main effect of sex with females completing sessions more quickly than males $\left(F_{1,61}=4.49, p<0.05\right.$; Table 2$)$. In addition, there was a significant effect of cocaine dose $\left(F_{1,22}=11.31, p<0.05\right)$, with food reinforcement sessions taking longer at the high cocaine dose. There was no interaction between sex and dose for session time during food reinforcement.

Concurrent reinforcement. Percent cocaine choice during concurrent reinforcement was analyzed via a $2 \times 2$ (sexdose) ANOVA, which revealed both a main effect of sex $\left(F_{1,79}=8.15, p<0.05\right)$ with females choosing cocaine over food more than males, and a significant effect of cocaine dose $\left(F_{1,81}=12.00, \quad p<0.05\right) \quad$ with significantly greater cocaine choice at the high dose of cocaine for both males and females (Figure 1a). During concurrent reinforcement, cocaine preference ( $>50 \%$ cocaine choice) was displayed in $58.6 \%$ (17 out of 29 ) of the females at the low dose of cocaine, and $76.47 \%$ (13 out of 17) of females at the high dose of cocaine. In males, cocaine preference was displayed in $33.33 \%$ (6 out of 18 ) of male subjects of the males at the low cocaine dose, and $68.42 \%$ (13 out of 16 ) of male subjects of the males at the high cocaine dose. Session time was 

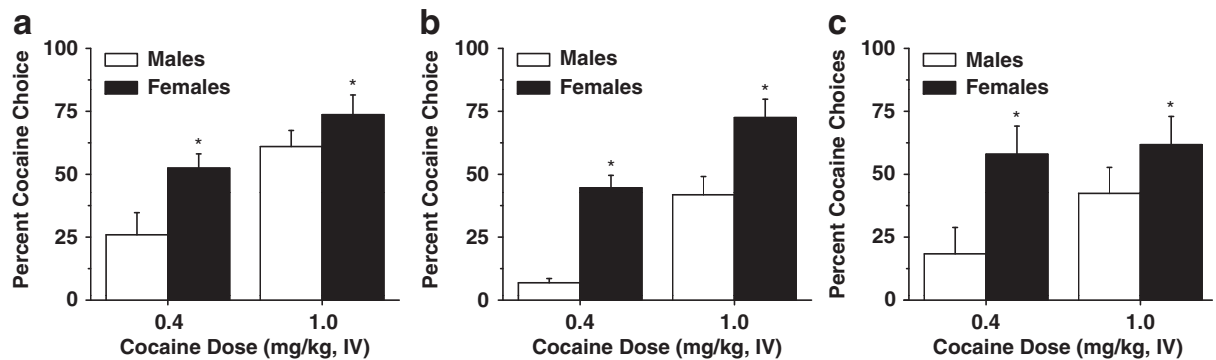

Figure I Impact of sex on cocaine choice during concurrent reinforcement, discrete trials, and reverse discrete trials under food restriction and sexspecific food reinforcement conditions. (a) Percent cocaine choice during concurrent reinforcement. Female rats choose cocaine over food more than males at both doses. (b) Percent cocaine choice during discrete trials. Female rats choose cocaine more often than males at both doses. (c) Percent cocaine choice during reverse discrete trials. Female rats choose cocaine more than males both doses. *Significant effect of sex, $p<0.05$.

analyzed via a $2 \times 2(\operatorname{sex} \times$ dose $)$ ANOVA. There was a significant effect of cocaine dose with sessions taking longer at the higher cocaine dose $\left(F_{1,61}=22.27, p<0.05\right.$; Table 2$)$. In addition, there was a significant effect of $\operatorname{sex}\left(F_{1,61}=4.63\right.$, $p<0.05$ ), with female rats taking longer than males to complete concurrent reinforcement sessions, which is likely related to their higher cocaine choice. No interaction between sex and dose was detected for session time during concurrent reinforcement sessions.

Further, responses during concurrent reinforcement were analyzed via a $2 \times 2 \times 2$ (lever $\times$ sex $\times$ dose) ANOVA, which revealed a significant three-way interaction $\left(F_{1,125}=7.97\right.$, $p<0.05)$. For the low dose of cocaine, a $2 \times 2($ lever $\times$ sex $)$ ANOVA revealed a sex $\times$ lever interaction $\left(F_{1,80}=15.09\right.$, $p<0.05$ ), with males responding more on the foodreinforced lever relative to females $(p<0.05$; Table 1$)$, but no sex differences were found for responding on the cocaine-reinforced lever. For the high dose of cocaine, $2 \times 2$ (lever $\times$ sex) ANOVA failed to reveal significant effects or interactions.

Discrete trials. Percent cocaine choice during discrete trials was analyzed via a $2 \times 2$ (sex $\times$ dose $)$ ANOVA, which revealed a significant effect of sex $\left(F_{1,79}=35.43, p<0.05\right)$, with females choosing cocaine significantly more than males at both cocaine doses, and a significant effect of cocaine dose $\left(F_{1,79}=29.90, p<0.05\right)$ with greater cocaine choice at the high dose of cocaine $(1.0 \mathrm{mg} / \mathrm{kg}$ per infusion) for both sexes (Figure 1b). No significant interaction between sex and dose was detected. During discrete trials, cocaine preference ( $>50 \%$ cocaine choice) was displayed in $37.04 \%$ (10 out of 27) of the females at the low dose of cocaine, and $82.35 \%$ (14 out of 18 ) of females at the high dose of cocaine. In males, cocaine preference was not displayed in any of the males at the low cocaine dose, and in $36.84 \%$ (7 out of 19 ) of the males, displayed a cocaine preference at the high cocaine dose. Further, session time (ie, time to receive 25 reinforcers) was analyzed via a $2 \times 2$ (sex $\times$ dose) ANOVA for discrete trial sessions, which revealed a significant effect of sex $\left(F_{1,56}=5.141, p<0.05\right.$; Table 2), with female rats taking longer than males to complete discrete trial sessions (which is likely related to their higher cocaine choice) and a significant effect of cocaine dose $\left(F_{1,56}=30.57, p<0.05\right)$ with sessions taking longer at the higher cocaine dose. No interaction between sex and dose was detected for session time during discrete trial sessions.
Table 3 Percent Cocaine Choice During Discrete Trial Sessions as a Function of Estrous Cycle (all Data Represents Mean \pm SEM)

\begin{tabular}{llcc}
\hline \multirow{2}{*}{ Cocaine dose (mg/kg, i.v.) } & \multicolumn{3}{c}{ Reproductive cycle phase } \\
\cline { 2 - 4 } & Diestrus & Proestrus & Estrus \\
\hline 0.4 & $48.6 \pm 6.0$ & $44.6 \pm 9.0$ & $44.1 \pm 4.1$ \\
1 & $75.6 \pm 15.9$ & $79.4 \pm 6.0$ & $75.2 \pm 14.1$ \\
\hline
\end{tabular}

Reverse discrete trials. Percent cocaine choice during reverse discrete choice trials was analyzed via a $2 \times 2$ $($ sex $\times$ dose) ANOVA, which revealed a significant sex effect $\left(F_{1,31}=6.70, p<0.05\right)$, with females choosing cocaine significantly more than males at both cocaine doses (Figure 1c). No interaction or main effect of cocaine dose was detected. This indicates that sex differences are maintained even under conditions of reversal learning, such that males continue to exhibit a higher preference for food reinforcement, and females continue to exhibit a higher preference for cocaine reinforcement.

Estrous cycle analysis. Given the evidence that the reproductive cycle modulates responsiveness to cocaine reinforcement during non-concurrent access experiments, we examined the relation of estrous cycle phase (diestrus: $n=15$, proestrus: $n=10$, estrus: $n=18$ ) to cocaine choice during the discrete trial phase of the above experiment. However, no interactions or main effects of estrous phase were detected (all $p>0.05$, Table 3 ).

\section{Food Reinforcement, Food Restriction, and ITI Manipulations}

To control for the potential contributions of differential size of food reinforcers or food restriction to the above findings, an additional set of rats were tested under the same conditions as above, with males receiving two food pellets as reinforcement, and another set of rats were tested under the same conditions as above, with ad libitum access to food. Further, to assess the possibility that sex differences in cocaine choice are due to increased immediate effects of cocaine (eg, anorexia) in females rats relative to males, another set of male and female rats were tested under the same conditions as above, except that an ITI of $10 \mathrm{~min}$ was imposed during the discrete trials phase (this time was 
chosen to reduce the immediate effects of cocaine impacting the next choice). Because of differences in acquisition at the low cocaine dose, the high cocaine dose $(1.0 \mathrm{mg} / \mathrm{kg}$ per infusion) was used for these experiments.

Males trained and tested with reinforcement of two food pellets, as well as with reinforcement of $1.0 \mathrm{mg} / \mathrm{kg}$ (i.v.) cocaine throughout the same sequence of experimental phases (ie, acquisition, concurrent reinforcement, and discrete choice), were compared with females receiving the same level of food and cocaine reinforcement. Sex differences were not detected during acquisition, lever responding during training, or lever responding on the cocaine lever under these conditions (all $p>0.05$; Table 1 ). There was an effect of sex on food lever responding during concurrent reinforcement with males pressing more than females $\left(F_{1,16}=4.20, \quad p<0.05\right.$; Table 1$)$, which is in agreement with the sex-specific food reinforcement results detected above. Moreover, females choose cocaine over food more than males during discrete trial phases of the experiment; one-way ANOVA of cocaine choice revealed a significant effect of sex $\left(F_{1,21}=6.80, p<0.05\right)$, with females choosing cocaine more than males (Figure $2 \mathrm{a}$ ). In addition, we compared cocaine choice during discrete trials in males who received two pellets $v s$ males who received three pellets, and found no effect of food reinforcement level on cocaine choice $(p>0.5)$. Thus, sex differences in cocaine choice observed above cannot be explained by differences in the magnitude of the food reinforcement.

To address the possibility that there were sex differences in food restriction that lead to sex differences in cocaine choice, the same procedures as above were also performed in intact females and males on ad libitum access to rat chow. There was no effect of sex on acquisition, lever responding during training, or lever responding during concurrent reinforcement under these conditions (Table 1). Moreover, a one-way ANOVA revealed a significant effect of sex $\left(F_{1,10}=54.68, p<0.05\right)$, with females choosing cocaine significantly more than males during discrete trials (Figure 2b). Thus, sex differences in cocaine choice observed above cannot be explained by differences in food restriction-induced hunger, because sex differences were also observed under ad libitum feeding conditions.

Finally, we examined cocaine choice with a longer ITI (10 min) during discrete trial sessions. A one-way ANOVA revealed that females exhibited higher cocaine choice than males $\left(F_{1,14}=4.62, p<0.05\right)$, with females choosing cocaine more than males (Figure 2c). Additionally, it was noticed that animals did not always earn the maximum number of reinforcers during these sessions; however, there were no sex differences in the total numbers of reinforcers earned during discrete trial sessions with the ITI of $10 \mathrm{~min}(p>0.05$; mean \pm SEM percent cocaine choice, for females $22.40 \pm 0.06$ and for males $23.00 \pm 0.44$ ). Notably, during these trials, cocaine choice was lower for both male and female rats than with a 20-s interval (compare with Figure 1b). These findings indicate that acute cocaine intoxication does impact cocaine choice, but does not appear to mediate sex differences.

\section{Impact of Ovariectomy and Estrogen Replacement on Cocaine Choice}

Separate groups of females were used to assess the impact of estrogen on cocaine choice. OVX females treated with either peanut oil (OVX + veh; $n=8)$ or EB (OVX + EB; $n=8)$ were compared with an additional group of intact females $(n=4)$ that did not undergo daily vaginal smears. All females were trained to respond for two food pellets ( $45 \mathrm{mg}$ each) and $1.0 \mathrm{mg} / \mathrm{kg}$ per infusion cocaine (this dose was chosen, because there were no sex differences during acquisition of operant behavior in the previous experiment).

Acquisition of operant responding. Neither OVX or hormone replacement had an impact on the number of days to meet the criteria for self-administration. The number of days to meet criterion for self-administration was analyzed via a one-way ANOVA that failed to reveal any significant differences between groups (mean \pm SEM for days to meet criteria, $13.02 \pm 0.42$ for intact; $12.50 \pm 0.26$ for OVX + veh; $12.59 \pm 0.30$ for OVX $+E B)$.

Cocaine and food reinforcement. Similarly, OVX and hormone replacement also failed to impact lever responding during acquisition of cocaine and food reinforcement phase. For cocaine reinforcement, the number of responses was analyzed via a one-way ANOVA, and this failed to reveal a significant difference $(p>0.05)$ between groups (mean \pm SEM for lever responses, $48.95 \pm 8.76$ for intact; $46.42 \pm 3.35$ for OVX + veh; $44.28 \pm 3.53$ for OVX + EB). For food reinforcement, the number of responses was analyzed via a one-way ANOVA, which failed to reveal a significant difference $(p>0.05)$ between groups (mean \pm SEM for lever responses, $98.65 \pm 20.09$ for intact; $90.69 \pm 11.73$ for OVX + veh; $78.86 \pm 9.04$ for OVX $+\mathrm{EB})$.
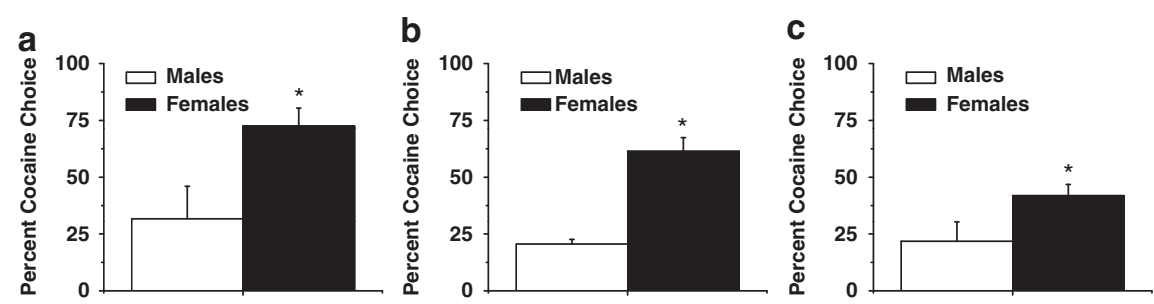

Figure 2 Impact of sex on cocaine choice during discrete trials under ad libitum feeding, equivalent food reinforcement value, and I0-min inter-trial interval conditions. (a) Percent cocaine choice during discrete trials with two-pellet reinforcement for both male and female; females choose cocaine significantly more than males. (b) Percent cocaine choice during discrete trials with ad libitum home-cage feeding, females choose cocaine significantly more than males. (c) Percent cocaine choice during discrete trials with 10-min inter-trial intervals, females choose cocaine significantly more than males. *Significant effect of sex, $p<0.05$. 

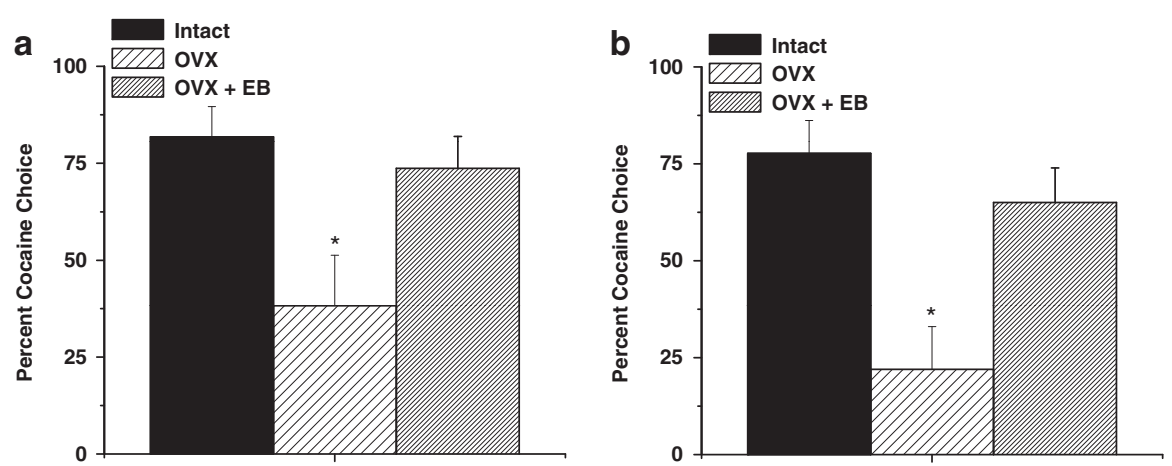

Figure 3 Impact of ovariectomization $(\mathrm{OVX})$ and estrogen replacement on choice between food and cocaine in female rats. OVX females exhibited fewer cocaine choices than intact females, or OVX females treated with estrogen benzoate (EB; $5 \mu \mathrm{g}$ per day) during concurrent reinforcement (a) and during discrete trial (b) sessions. *Significant effect relative to intact female, $p<0.05$.

Concurrent reinforcement. Intact and $\mathrm{OVX}+\mathrm{EB}$, but not OVX + veh, females exhibited a preference for cocaine over food reinforcement. Percent cocaine choice was analyzed via a one-way ANOVA, which revealed an effect of hormones status $\left(F_{2,16}=3.63, p=0.05\right)$, with intact and OVX $+\mathrm{EB}$ females displaying greater cocaine choice than OVX + veh (Figure 3a). Responses during concurrent reinforcement were analyzed via a $2 \times 3$ (lever $\times$ hormone status) ANOVA; no interactions or main effects of lever or hormone status were detected.

Discrete trials. Similarly, during discrete trial sessions, intact and OVX + EB, but not OVX + veh, females continued to display a preference for cocaine over food reinforcement. Percent cocaine choice was analyzed using a one-way ANOVA, which revealed a significant effect of group $\left(F_{2,16}=9.30, \quad p<0.05\right.$; Figure $\left.3 \mathrm{~b}\right)$. Bonferroni post hoc analysis revealed significant differences between intact and OVX + EB females relative to OVX + veh females $(p s<0.05)$, with intact and OVX $+\mathrm{EB}$ females choosing cocaine to a greater extent than OVX + veh females during discrete trials. These data indicate that ovarian hormones are critical for placing a higher preference on cocaine in females during a discrete trial procedure.

\section{DISCUSSION}

The main finding of the present study is that female rats choose cocaine over food significantly more often than males. The present study provides the first examination of the effects of sex, ovariectomy, and estrogen on choice between cocaine and food. During discrete trials, at the low dose of cocaine, males exhibit a clear preference for food reinforcement, whereas females respond for food and cocaine at an approximately equal level. At the higher dose of cocaine, males maintain a food preference, whereas females display a cocaine preference. Furthermore, both sexes were able to track reinforcers when the lever reinforcer was reversed, indicating that the behavior was directed at obtaining a specific reinforcer rather than habitual responding. The observed sex differences are dependent upon female gonadal hormones, as OVX females displayed a preference for food over cocaine, and OVX females treated with estrogen displayed a preference for cocaine over food, which was similar to that observed in intact females. However, cocaine choice in females was not linked to reproductive cycle status, suggesting that it is likely independent of hormone levels at the time of testing, but rather depends on other effects of ovarian hormones.

It was also found that female rats take longer than males to complete discrete trial sessions, which is likely related to their higher cocaine choice. This suggests that higher cocaine choice in females may depend on their ability to space their reinforcement out over a longer period, indicating that higher cocaine choice in females is not explained by higher impulsivity exhibited by female rats when different amounts of a single type of reinforcer is available (ie, Anker et al, 2008; Perry et al, 2007). Given that maladaptive decisionmaking contributes to drug dependence in humans (American Psychiatric Association (1994); Santolaria-Fernández et al, 1995; van Etten et al, 1998), the present findings indicate that there is a biological predisposition for females to forgo food reinforcement to obtain cocaine reinforcement, which may substantially contribute to women experiencing a more severe profile of cocaine addiction than men.

Interestingly, food restriction appeared to reduce the proportion of food reinforcers selected in both sexes (ie, $\sim 22 \%$ decrease food choice in males and $\sim 11 \%$ for females). It was somewhat unexpected that food restriction would decrease food choice and shift selection toward cocaine, and this is in contrast to the effects observed for the impact of food restriction of the levels of responding for food reinforcement under single reinforcer conditions (Heinsbroek et al, 1987; van Haaren et al, 1989; Fulton et al, 2002). However, this change is consistent with the ability of food restriction to increase cocaine intake during cocaine self-administration (see Carroll et al, 1981; Campbell and Carroll, 2001), suggesting that the cocaine motivational effects of food restriction under these conditions are greater than the food motivational effects. Importantly, the observed sex differences in cocaine choice are not attributable to food reinforcement level, because cocaine choice did not change significantly when male rats selected between cocaine, and either two or three pellets of food. In contrast, studies in non-human primates have demonstrated that cocaine choice is sensitive to the level of 
the alternative reinforcer (eg, Nader and Woolverton, 1991). Still, it is likely that shifts in cocaine choice exhibited by rats would be sensitive to adjustments in level of food reinforcement that are of greater magnitude than those employed here. Nevertheless, the observed sex differences in motivation for the food reinforcer induced by food restriction or magnitude of reinforcer cannot explain sex differences in cocaine choice behavior under these conditions.

When extending the ITI from $20 \mathrm{~s}$ to $10 \mathrm{~min}$ under discrete trial conditions, sex differences were maintained with females choosing cocaine more than males. This indicates that the immediate effects of cocaine cannot explain sex differences in cocaine choice. However, both male and female rats decreased their cocaine choice when the ITI was extended, suggesting that cocaine intoxication enhances cocaine choice in both sexes. It is still possible that cocaine intoxication lasts longer in females than males, and if the ITI is extended beyond $10 \mathrm{~min}$, sex differences in cocaine choice may be abolished. As such, additional studies should be conducted to assess the impact of ITI duration on cocaine choice in male and female rats. Notably, the observed level of cocaine choice in males tested under discrete trial conditions with an ITI of $10 \mathrm{~min}$ is comparable to the findings of Lenoir et al (2007), who report $25 \%$ or less cocaine choice (days 6-15) in male rats during discrete trials with 10 -min intervals and employed a forced choice between a $0.25 \mathrm{mg}$ (i.v.) cocaine reinforcer (in rats weighing between 221 and $276 \mathrm{~g}$, which is approximately a $1.0 \mathrm{mg} / \mathrm{kg}$ i.v. dose) $v s$ a sweetened water reinforcer. This suggests that grain pellets and sweetened water are of approximately equal value as an alternative reinforcer compared with cocaine for male rats.

One possible reason for the increased cocaine choice in females, relative to males, may be sex differences in the propensity to perform a food- and cocaine-reinforced response. In the present study, sex differences were detected in acquisition for operant behavior at the low, but not the high, cocaine dose. In addition, there were sex differences in lever responding during cocaine and food reinforcement training, with males pressing more for both reinforcers when the cocaine dose was low, and females pressing more for both reinforcers when the cocaine dose was high. Despite the difference in acquisition and the opposite direction of the sex differences between cocaine doses during food and cocaine reinforcement, the same effect of sex was found during discrete trials, with females choosing cocaine significantly more than males at both cocaine doses. This suggests that despite sex differences in rate of responding, the increased cocaine choice in females, relative to males, is maintained. Further, the preference for food or cocaine in males and females were also maintained when the lever-reinforcer relation was reversed, indicating that habitual responding on a specific lever cannot account for the sex differences in cocaine choice. Accordingly, these findings indicate that the sex difference in cocaine choice is not merely a reflection of sex differences in response rates for food and cocaine.

The finding that female rats choose cocaine more frequently contrasts with reports that females show a greater reduction in drug intake when another reinforcer is present. Specifically, female primates will reduce their intake of PCP more significantly than males when they are given sucrose concurrently (Cosgrove and Carroll, 2003), and female rats will reduce cocaine intake significantly more than male rats when a running wheel is made available during cocaine self-administration sessions (Cosgrove et al, 2002). An important distinction is that these studies did not assess choice behavior with discrete trials, but rather used a concurrent access procedure in which both reinforcers were freely available throughout the session without a limit on the total reinforcers earned during a session, and in the case of concurrent wheel access, without an imposed operant necessary to gain access to the wheel. Accordingly, a concurrent reinforcement procedure under discrete trial conditions reveals a higher value of cocaine exhibited by females relative to males.

The relation between cocaine choice and ovarian hormones in females appears to be complex. Although ovaries or estrogen replacement are necessary for high cocaine choice in females, no effects of estrous were observed for cocaine choice. This suggests that the long-term effects of ovarian hormones influence cocaine choice. The dependence of cocaine choice on intact ovaries in females is generally consistent with evidence, indicating that ovariectomy reduces cocaine self-administration acquisition ( $\mathrm{Hu}$ and Becker, 2008; Jackson et al, 2006; Lynch et al, 2001), cocaine intake (Zhao and Becker, 2010; Larson et al, 2007), and cocaineseeking behavior (Anker et al, 2007). Interestingly, ovariectomy and the estrous cycle have been shown to impact food consumption consistently (Asarian and Geary, 2006; Thammacharoen et al, 2009; Santollo and Eckel, 2009; Wallen et al, 2001), whereas the evidence is less clear for responding during food reinforcement, such that OVX does not always reduce food reinforced responding (van Hest et al, 1989; Heinsbroek et al, 1987). This suggests that the impact of OVX on cocaine choice may be more related to OVX impacting the preference for cocaine, rather than food, reinforcement. However, the lack of estrous cycle effect on cocaine choice contrasts with the ability of estrous cycle phase to modulate lever responding during cocaine self-administration (Roberts et al, 1989; Kerstetter et al, 2008) and reinstatement of cocaine-seeking behavior (Feltenstein and See, 2007; Kerstetter et al, 2008; Kippin et al, 2005). Collectively, these data indicate that different biological processes than those mediating rate-dependent effects observed in single reinforcer studies mediate cocaine choice in females. Further, the present finding that estrogen administration increases cocaine choice in OVX females is in agreement with studies reporting that estrogen enhances the reinforcing properties of cocaine (Lynch et al, 2001; Larson et al, 2007; Zhao and Becker, 2010). The ability of estrogen to increase the preference for cocaine in females may be to due to estrogenregulating dopamine transmission, as estrogen increases dopamine turnover, regulates dopamine receptor (D1R) calcium/calmodulin-dependent protein kinase II activity, and alters dopamine neural firing in the mesolimbic dopamine system in female rats (Shieh and Yang, 2008; Zhang et al, 2008; Zhen et al, 2007). Accordingly, it would be of interest to investigate the potential of dopaminergic mechanism in mediating cocaine choice, as distinct from those increasing response rates in rats.

The observed sex differences in cocaine choice may be relevant to the clinical differences seen in men and women regarding nutritional status. Santolaria-Fernández et al 
(1995) found an effect of sex in the degree of malnutrition in a group of heroin- and cocaine-dependent subjects, with females displaying poorer nutritional status than males. Thus, similar to female rats, drug-experienced women appear to devalue food (and perhaps other natural reinforcers) relative to taking drugs to a greater extent than men, which is likely a significant contributor to the more severe profile of cocaine dependence that women display. Future projects are required to assess the role of estrogen in cocaine choice in a more detailed fashion, as well as examine the effect of other ovarian hormones (eg, progesterone and its metabolite, allopregnanolone) on cocaine choice, as this ovarian hormone has been shown to decrease motivation for cocaine in single reinforcer studies (Feltenstein et al, 2009; Anker et al, 2009).

\section{ACKNOWLEDGEMENTS}

This work was supported by NIDA Grants DA027525 and DA027115, and a NARSAD Young Investigator Awards to TEK.

\section{DISCLOSURE}

The authors declare no conflict of interest.

\section{REFERENCES}

Ahmed SH (2010). Validation crisis in animal models of drug addiction: beyond non-disordered drug use toward drug addiction. Neurosci Biobehav Rev 35: 172-184.

American Psychiatric Association (1994). Diagnostic and Statistical Manual of Mental Disorders, 4th edn. Washington, DC: Author.

Anker JJ, Carroll ME (2010). The role of progestins in the behavioral effects of cocaine and other drugs of abuse: human and animal research. Neurosci Biobehav Rev 35: 315-333.

Anker JJ, Gliddon LA, Carroll ME (2008). Impulsivity on a Go/Nogo task for intravenous cocaine or food in male and female rats selectively bred for high and low saccharin intake. Behav Pharmacol 19: 615-629.

Anker JJ, Holtz NA, Zlebnik N, Carroll ME (2009). Effects of allopregnanolone on the reinstatement of cocaine-seeking behavior in male and female rats. Psychopharmacology 203: 63-72.

Anker JJ, Larson EB, Gliddon LA, Carroll ME (2007). Effects of progesterone on the reinstatement of cocaine-seeking behavior in female rats. Exp Clin Psychopharmacol 15: 472-480.

Asarian L, Geary N (2006). Modulation of appetite by gonadal steroid hormones. Philos Trans $R$ Soc Lond B Biol Sci 361: 1251-1263.

Back SE, Brady KT, Jackson JL, Salstrom S, Zinzow H (2005). Gender differences in stress reactivity among cocaine-dependent individuals. Psychopharmacology 180: 169-176.

Banks ML, Gould RW, Czoty PW, Nader MA (2008). Relationship between response rates and measures of reinforcing strength using a choice procedure in monkeys. Behav Pharmacol 19: 365-369.

Banks ML, Negus SS (2010). Effects of extended cocaine access and cocaine withdrawal on choice between cocaine and food in rhesus monkeys. Neuropsychopharmacology 35: 493-504.

Becker JB (2009). Sexual differentiation of motivation: a novel mechanism? Horm Behav 55: 646-654.

Caine SB, Bowen CA, Yu G, Zuzga D, Negus SS, Mello NK (2004). Effect of gonadectomy and gonadal hormone replacement on cocaine self-administration in female and male rats. Neuropsychopharmacology 29: 929-942.
Campbell UC, Carroll ME (2001). Effects of ketoconazole on the acquisition of intravenous cocaine self-administration under different feeding conditions in rats. Psychopharmacology 154: 311-318.

Cantin L, Lenoir M, Augier E, Vanhille N, Dubreucq S, Serre F et al (2010). Cocaine is low on the value ladder of rats: possible evidence for resilience to addiction. PLoS One 5: e11592.

Carroll ME, France CP, Meisch RA (1981). Intravenous selfadministration of etonitazene, cocaine and phencyclidine in rats during food deprivation and satiation. J Pharmacol Exp Ther 217: 241-247.

Cosgrove KP, Carroll ME (2003). Effects of a non-drug reinforcer, saccharin, on oral self-administration of phencyclidine in male and female rhesus monkeys. Psychopharmacology 170: 9-16.

Cosgrove KP, Hunter RG, Carroll ME (2002). Wheel-running attenuates intravenous cocaine self-administration in rats: sex differences. Pharmacol Biochem Behav 73: 663-671.

Donny EC, Bigelow GE, Walsh SL (2003). Choosing to take cocaine in the human laboratory: effects of cocaine dose, inter-choice interval, and magnitude of alternative reinforcement. Drug Alcohol Depend 69: 289-301.

Evans SM, Foltin RW (2006). Exogenous progesterone attenuates the subjective effects of smoked cocaine in women, but not in men. Neuropsychopharmacology 31: 659-674.

Evans SM, Haney M, Foltin RW (2002). The effects of smoked cocaine during the follicular and luteal phases of the menstrual cycle in women. Psychopharmacology 159: 397-406.

Feltenstein MW, Byrd EA, Henderson AR, See RE (2009). Attenuation of cocaine-seeking by progesterone treatment in female rats. Psychoneuroendocrinology 34: 343-352.

Feltenstein MW, See RE (2007). Plasma progesterone levels and cocaine-seeking in freely cycling female rats across the estrous cycle. Drug Alcohol Depend 89: 183-189.

Fuchs RA, Evans KA, Mehta RH, Case JM, See RE (2005). Influence of sex and estrous cyclicity on conditioned cue-induced reinstatement of cocaine-seeking behavior in rats. Psychopharmacology 179: 662-672.

Fulton S, Woodside B, Shizgal P (2002). Does neuropeptide Y contribute to the modulation of brain stimulation reward by chronic food restriction? Behav Brain Res 134: 157-164.

Heinsbroek RP, van Haaren F, Zantvoord F, van de Poll NE (1987). Sex differences in response rates during random ratio acquisition: effects of gonadectomy. Physiol Behav 39: 269-272.

Higgins ST, Bickel WK, Hughes JR (1994). Influence of an alternative reinforcer on human cocaine self-administration. Life Sci 55: 179-187.

Hu M, Becker JB (2008). Acquisition of cocaine self-administration in ovariectomized female rats: effect of estradiol dose or chronic estradiol administration. Drug Alcohol Depend 94: 56-62.

Jackson LR, Robinson TE, Becker JB (2006). Sex differences and hormonal influences on acquisition of cocaine self-administration in rats. Neuropsychopharmacology 31: 129-138.

Katz JL (1990). Models of relative reinforcing efficacy of drugs and their predictive utility. Behav Pharmacol 1: 283-301.

Kerstetter KA, Aguilar VR, Parrish AB, Kippin TE (2008). Protracted time-dependent increases in cocaine-seeking behavior during cocaine withdrawal in female relative to male rats. Psychopharmacology 198: 63-75.

Kerstetter KA, Chitaya LD, Reyes M, Chenpanas P, Kippin TE (2008b). Estrous cycle modulation of cocaine-primed reinstatement of food-seeking behavior in female rats. Soc Neurosci, published online on Nov 2008 at abstractsonline.com/Plan/ SSResults.aspx.

Kippin TE, Fuchs RA, Mehta RH, Case JM, Parker MP, BimonteNelson HA et al (2005). Potentiation of cocaine-primed reinstatement of drug seeking in female rats during estrus. Psychopharmacology 182: 245-252. 
Kosten TA, Gawin FH, Kosten TR, Rounsaville BJ (1993). Gender differences in cocaine use and treatment response. J Subst Abuse Treat 10: 63-66.

Larson EB, Anker JJ, Gliddon LA, Fons KS, Carroll ME (2007). Effects of estrogen and progesterone on the escalation of cocaine self-administration in female rats during extended access. Exp Clin Psychopharmacol 15: 461-471.

Lenoir M, Serre F, Cantin L, Ahmed SH (2007). Intense sweetness surpasses cocaine reward. PLoS One 2: e698.

Lynch WJ, Carroll ME (1999). Sex differences in the acquisition of intravenously self-administered cocaine and heroin in rats. Psychopharmacology 144: 77-82.

Lynch WJ, Roth ME, Mickelberg JL, Carroll ME (2001). Role of estrogen in the acquisition of intravenously self-administered cocaine in female rats. Pharmacol Biochem Behav 68: 641-646.

Lynch WJ, Taylor JR (2004). Sex differences in the behavioral effects of 24-h/day access to cocaine under a discrete trial procedure. Neuropsychopharmacology 29: 943-951.

Nader MA, Woolverton WL (1991). Effects of increasing the magnitude of an alternative reinforcer on drug choice in a discrete-trials choice procedure. Psychopharmacology (Berl) 105: 169-174.

Negus SS (2003). Rapid assessment of choice between cocaine and food in rhesus monkeys: effects of environmental manipulations and treatment with d-amphetamine and flupenthixol. Neuropsychopharmacology 28: 919-931.

Perry JL, Nelson SE, Anderson MM, Morgan AD, Carroll ME (2007). Impulsivity (delay discounting) for food and cocaine in male and female rats selectively bred for high and low saccharin intake. Pharmacol Biochem Behav 86: 822-837.

Roth ME, Carroll ME (2004). Sex differences in the escalation of intravenous cocaine intake following long- or short-access to cocaine self-administration. Pharmacol Biochem Behav 78: 199-207.

Roberts DC, Bennett SA, Vickers GJ (1989). The estrous cycle affects cocaine self-administration on a progressive ratio schedule in rats. Psychopharmacology 98: 408-411.

Santolaria-Fernández FJ, Gómez-Sirvent JL, González-Reimers CE, Batista-López JN, Jorge-Hernández JA, Rodríguez-Moreno F et al (1995). Nutritional assessment of drug addicts. Drug Alcohol Depend 38: 11-18.

Santollo J, Eckel LA (2009). Effect of a putative ERalpha antagonist, MPP, on food intake in cycling and ovariectomized rats. Physiol Behav 97: 193-198.
Sofuoglu M, Dudish-Poulsen S, Nelson D, Pentel PR, Hatsukami DK (1999). Sex and menstrual cycle differences in the subjective effects from smoked cocaine in humans. Exp Clin Psychopharmacol 7: 274-283.

Shieh KR, Yang SC (2008). Effects of estradiol on the stimulation of dopamine turnover in mesolimbic and nigrostriatal systems by cocaine- and amphetamine-regulated transcript peptide in female rats. Neuroscience 154: 1589-1597.

Thammacharoen S, Geary N, Lutz TA, Ogawa S, Asarian L (2009). Divergent effects of estradiol and the estrogen receptoralpha agonist PPT on eating and activation of PVN CRH neurons in ovariectomized rats and mice. Brain Res 1268: 88-96.

van Etten ML, Higgins ST, Budney AJ, Badger GJ (1998). Comparison of the frequency and enjoyability of pleasant events in cocaine abusers vs. non-abusers using a standardized behavioral inventory. Addiction 93: 1669-1680.

van Haaren F, van Hest A, van Hattum T (1989). Scopolamine and methylscopolamine differentially affect fixed-consecutive-number performance of male and female Wistar rats. Pharmacol Biochem Behav 33: 361-365.

van Hest A, van Haaren F, van de Poll NE (1989). Perseverative responding in male and female Wistar rats: effects of gonadal hormones. Horm Behav 23: 57-67.

Wallen WJ, Belanger MP, Wittnich C (2001). Sex hormones and the selective estrogen receptor modulator tamoxifen modulate weekly body weights and food intakes in adolescent and adult rats. J Nutr 131: 2351-2357.

Westermeyer J, Boedicker AE (2000). Course, severity, and treatment of substance abuse among women versus men. Am J Drug Alcohol Abuse 26: 523-535.

Zhang D, Yang S, Yang C, Jin G, Zhen X (2008). Estrogen regulates responses of dopamine neurons in the ventral tegmental area to cocaine. Psychopharmacology 199: 625-635.

Zhao W, Becker JB (2010). Sensitization enhances acquisition of cocaine self-administration in female rats: estradiol further enhances cocaine intake after acquisition. Horm Behav 58: 8-12.

Zhen X, Goswami S, Abdali SA, Frankfurt M, Friedman E (2007). Estrogen-modulated frontal cortical CaMKII activity and behavioral supersensitization induced by prolonged cocaine treatment in female rats. Psychopharmacology 191: 323-331. 